\title{
Pattern of Recruitment of Immunoregulatory Antigen-Presenting Cells in Malignant Melanoma
}

\author{
Jeffrey R. Lee, Rory R. Dalton, Jane L. Messina, Madhav D. Sharma, \\ David M. Smith, Russell E. Burgess, Fermina Mazzella, Scott J. Antonia, \\ Andrew L. Mellor, and David H. Munn
}

Institute of Molecular Medicine and Genetics (JRL, MDS, ALM, DHM), Augusta Veterans Affairs Medical Center (JRL), and Departments of Pathology (JRL, RRD, FM), Biostatistics and Bioinformatics (DMS), Medicine (REB, ALM), and Pediatrics (DHM), Medical College of Georgia, Augusta, Georgia; and Department of Pathology (JLM) and Interdisciplinary Oncology Program (SJA), H. Lee Moffitt Cancer Center, Tampa, Florida

SUMMARY: The mechanism by which the immune system of a tumor-bearing host acquires tolerance toward tumor antigens is still elusive. Antigen-presenting cells (APCs) are critical regulators of the decision between immune response and tolerance. APCs that express the tryptophan-degrading enzyme indoleamine 2,3-dioxygenase (IDO) have been found to inhibit T-cell responses both in vitro and in vivo. We hypothesized that malignant tumors exploit this mechanism by recruiting IDO-expressing APCs to the tumor-draining lymph nodes. To test this hypothesis, archival tissues and records of 26 cases of lymph node dissection for invasive cutaneous melanoma were obtained. IDO immunohistochemistry was performed on 14 cutaneous tumors and 328 regional lymph nodes. Abnormal accumulations of IDO-positive cells with a monocytoid or plasmacytoid morphology were identified in the perisinusoidal regions of draining lymph nodes in $45 \%$ of nodes studied. Recruitment of IDO-positive cells was seen in nodes with and without malignancy. We hypothesize that these IDO-positive APCs may contribute mechanistically to acquired tolerance to tumor antigens. Immunostaining of tumor-draining lymph nodes for abnormal accumulation of IDOexpressing cells might thus constitute an adverse prognostic factor and could contribute to the decision process and the appropriate care of patients with this deadly disease. (Lab Invest 2003, 83:1457-1466).

$T$ he ability of tumors to elude the host immune system is key to neoplastic growth and survival. Overcoming this aberrant tumor antigen tolerance has long been proposed as an important adjunct in oncologic therapeutics. Host immune tolerance is functional in the management of self- and tumorassociated antigens, and the efficiency of this process has thwarted the success of many human immunotherapy strategies (Houghton et al, 2001). Recent murine advances in the breaking of selftolerance including modified cross-reacting antigens (Bowne et al, 1999), enhanced dendritic cell (DC) presentation of tumor antigens (Klein et al, 2000), addition of helper epitopes (Shimizu et al, 2001), and use of potent adjuvants have been of limited value in treating human tumors.

There is increasing evidence that antigen presentation by certain bone marrow-derived antigenpresenting cells (APCs) is an important initiator of

\section{DOI: 10.1097/01.LAB.0000090158.68852.D1}

Received June 6, 2003.

Grant support: NIH RO1 HL60137 (to DHM) and the Carlos and Marguerite Mason Trust (to JRL, ALM, and DHM).

Address reprint requests to: Dr. Jeffrey $R$. Lee, Pathology and Laboratory Medicine Department, Veterans Affairs Medical Center, PLMD (239), One Freedom Way, Augusta, Georgia 30904. E-mail: JLEE@mail. mcg.edu immunologic self-tolerance (Albert et al, 2001; Dhodapkar et al, 2001; Doan et al, 2000; Jonuleit et al, 2001; Pardoll, 2001; Sotomayor et al, 2001; Steinman and Nussenzwieg, 2002). The characterization of these cells and the mechanism of in vivo tolerance induction, however, is still elusive. Recently, we proposed that the nodal presence of a subset of APCs expressing the tryptophan-catabolizing enzyme indoleamine 2,3-dioxygenase (IDO) was involved in the generation of host immunotolerance toward malignancy (Munn et al, 2002). Enzymatic activity of IDO has been reported in human monocyte-derived macrophages (Munn et al, 1999), human DCs (Hwu et al, 2000), murine CD8 $\alpha^{+}$DCs (Grohmann et al, 2001), and activated $\mathrm{CD}^{+}{ }^{+} \mathrm{T}$ cells (Curreli et al, 2001). Its expression by antigen-presenting cells has been shown to inhibit cell cycle progression and proliferation of $\mathrm{T}$ cells in vitro ( $\mathrm{Hwu}$ et al, 2000; Mellor et al, 2002; Munn et al, 1999). In vivo, IDO-expressing CD $8 \alpha^{+}$murine DCs induced tolerance in mature T cells (Grohmann et al, 2001), and in a pregnant mouse model, it has been suggested that IDO activity acts as an immunosuppressive barrier, protecting paternal antigen-bearing embryos from maternal T-cell immunity (Munn et al, 1998). Recently, IDO has been implicated as a downstream effector mechanism in tolerance induced by CTLA-4-Ig (Grohmann et al, 2002). Additionally, in vivo experiments using IDO transfected tumor cells and IDO transgenic mice dem- 
onstrated that the presence of transfected IDO was able to inhibit specific CD8 ${ }^{+}$responder cells (Mellor et al, 2002).

In vitro characterization of the previously reported IDO-positive human APCs showed that they expressed the myeloid lineage marker CD11c as well as CD123, CD83, CD80, CD86, and the chemokine receptor CCR6 (Munn et al, 2002), thus placing them in the myeloid DC lineage. Functionally, these cells were potent inhibitors of proliferation in allogeneic mixedleukocyte reactions. This inhibitory effect was reversed with the addition of 1-methyl-tryptophan, a competitive inhibitor of IDO. In vivo, accumulations of IDO-positive cells with a monocytoid or plasmacytoid morphology were also identified in chronically infected human tonsils and in lymph nodes from patients with melanoma and breast, colon, lung, and pancreatic carcinomas (Munn et al, 2002). Based on these observations, we hypothesized that IDO-positive cells were recruited to lymph nodes that drain human malignancies, where they may contribute to establishing immunologic unresponsiveness toward future metastatic disease. In the current study, we characterize the IDO-expressing cell population in draining lymph nodes of malignant melanoma.

\section{Results}

\section{Flow Cytometric Analysis of Monocyte-Derived IDO-Positive DCs}

Figure 1 demonstrates the characteristics of mature IDO-positive DCs derived by culturing peripheralblood monocytes in granulocyte-macrophage colonystimulating factor + IL-4 (Munn et al, 2002). These cells co-express CD123, CD11c, CD11b, CD80, CD83, and CD86, consistent with a phenotype of mature myeloid DCs. They demonstrated no detectable BDCA-2 expression, a marker of lymphoid DCs (pre-DC2). Essentially all IDO-positive and CD123- positive DCs co-expressed the chemokine receptor CCR6, even after maturation.

\section{Clinical Features of Patients with Melanoma}

The patient cohort (Table 1) consisted of 18 men and 8 women with an age range from 26 to 76 years (mean, 49 years). Clinical information was available on the primary cutaneous lesion in 25 patients; one patient had an unknown primary. The depth of invasion was available on all known primary melanomas, ranging from $0.97 \mathrm{~mm}$ to $15 \mathrm{~mm}$. Epidermal ulceration was present in 4 patients, absent in 12 patients, and information was unavailable on 10 patients.

Ten patients underwent removal of sentinel nodes, with one to four nodes (mean, 2.1 nodes) being retrieved from each dissection. Twenty patients underwent radical lymph node dissection, four of these following the discovery of positive sentinel nodes. A mean of 15.5 nodes were obtained from these dissections ( 7 to 35 nodes). Eleven patients had nodes that were negative for malignancy (including six negative sentinel node biopsies), four patients had one node positive for melanoma, seven patients had two to three positive nodes, and four patients had four or greater positive nodes. According to the updated American Joint Committee on Cancer staging system for melanoma, 15 patients harbored stage-III disease, 9 patients had stage-II disease, and 2 patients had stage-l (Balch et al, 2001) (Table 1).

Follow-up data were available on 26 patients, with a range of 7 to 73 months (mean, 28.7 months). Six patients died of melanoma at 7 to 53 months (mean, 28.8 months), while two patients developed concomitant pulmonary small cell carcinoma and died of undefined metastatic disease. One patient died without evidence of melanoma. Three patients were alive with disease at 16 to 54 months follow-up (mean, 30.8 months). Fourteen patients were free of disease at their last visit (9 to 73 months; mean, 23.5 months).
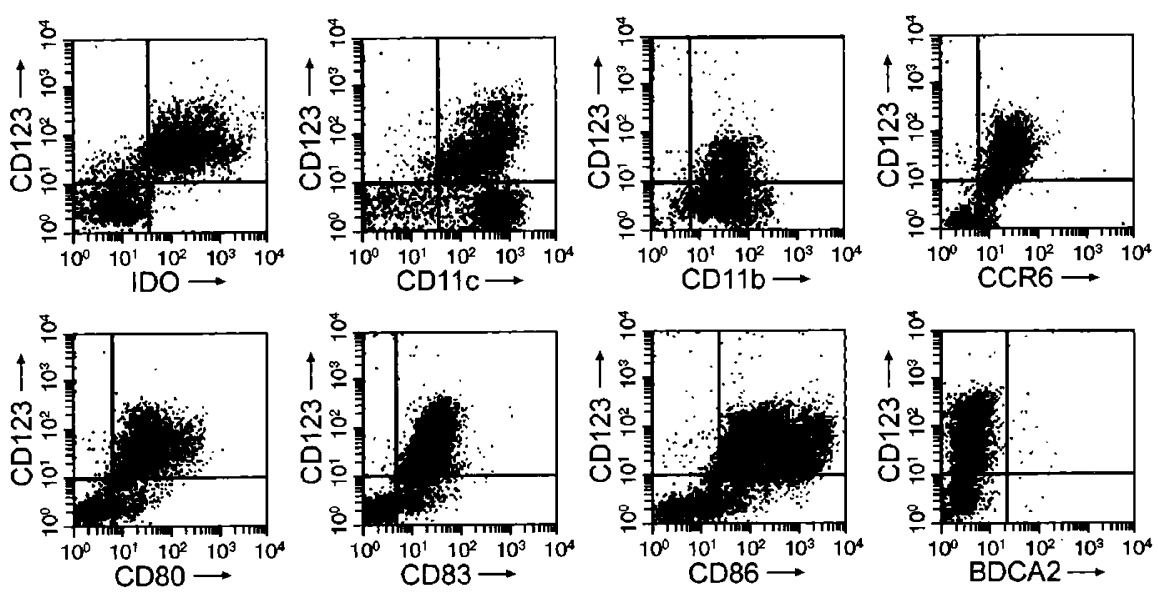

Figure 1.

Immunophenotype of in vivo macrophage derived dendritic cells. The first panel shows intracellular staining for ID0 to establish that all CD123-positive cells express intracellular indoleamine 2,3-dioxygenase (ID0), and that all IDO-positive cells express CD123. Subsequent panels use two-color cell surface immunophenotyping to show the pattern of markers on the CD123-positive subset. CD123-positive cells co-express CD11c, CD11b, CCR6, CD80, CD83, and CD86, and demonstrate no expression of BDCA-2. Representative of eight experiments. 
Table 1. Characteristics of Patient Cohort

\begin{tabular}{|c|c|c|c|c|c|c|c|c|}
\hline $\begin{array}{c}\text { Patient } \\
\text { no. }\end{array}$ & Age/sex & Stage & TNM & $\begin{array}{c}\text { Total } \\
\text { nodes/SNT }\end{array}$ & $\begin{array}{c}\text { Positive } \\
\text { nodes/SNP }\end{array}$ & IDO NH & IDO NS & Outcome \\
\hline 1 & $42 / F$ & IIIC & T4bN3M0 & $35 / 0$ & $14 / 0$ & $3+$ & 0.76 & ANED/21 mos \\
\hline 2 & $53 / F$ & IIIC & T4aN3MO & $7 / 0$ & $1 / 0(\mathrm{~s})$ & $N$ & 0.00 & AWD/16 mos \\
\hline 3 & $60 / \mathrm{M}$ & IIIC & T4bN3M0 & $17 / 0$ & $7 / 0$ & $2+$ & 0.43 & ANED/73 mos \\
\hline 4 & $63 / \mathrm{M}$ & IIIC & T3aN3M0 & $20 / 0$ & $5 / 0$ & $4+$ & 2.24 & $\mathrm{DOD} / 22 \mathrm{mos}$ \\
\hline 5 & $75 / \mathrm{M}$ & IIIC & T3aN3M0 & $13 / 3$ & $7 / 3$ & $4+$ & 1.92 & ANED/31 mos \\
\hline 6 & $42 / F$ & IIIC & T2xN3M0 & $19 / 1$ & $1 / 1(\mathrm{~s})$ & $4+$ & 0.37 & ANED/22 mos \\
\hline 7 & $51 / \mathrm{M}$ & IIIC & TxN3M0 & $7 / 1$ & $4 / 0$ & $1+$ & 0.50 & AWD/29 mos \\
\hline 8 & $62 / M$ & IIIB & T4aN2bM0 & $8 / 0$ & $2 / 0$ & $4+$ & 2.31 & DUD/7 mos \\
\hline 9 & $68 / \mathrm{M}$ & IIIB & T3aN2bM0 & $13 / 0$ & $2 / 0$ & $4+$ & 2.41 & DOD/31 mos \\
\hline 10 & $37 / F$ & IIIA & T4aN2aM0 & $18 / 1$ & $3 / 1$ & $1+$ & 1.62 & $\mathrm{DOD} / 17 \mathrm{mos}$ \\
\hline 11 & $59 / \mathrm{M}$ & IIIX & T3xN2bM0 & $13 / 0$ & $2 / 0$ & $\mathrm{~N}$ & 0.00 & DOD/20 mos \\
\hline 12 & $40 / \mathrm{M}$ & IIIX & T2xN2bM0 & $14 / 0$ & $2 / 0$ & $4+$ & 1.38 & ANED/45 mos \\
\hline 13 & $45 / \mathrm{M}$ & IIIX & T2xN2bM0 & $21 / 0$ & $3 / 0$ & $1+$ & 0.30 & ANED/10 mos \\
\hline 14 & $44 / F$ & IIIX & T4xN1bM0 & $24 / 0$ & $1 / 0$ & $1+$ & 0.28 & DFOD/51 mos \\
\hline 15 & 29/M & IIIX & T3xN1bM0 & $8 / 0$ & $1 / 0$ & $4+$ & 1.38 & ANED/7 mos \\
\hline 16 & $45 / \mathrm{M}$ & IIC & T4bNOMO & $2 / 2$ & $0 / 0$ & $2+$ & 1.00 & ANED/9 mos \\
\hline 17 & $30 / \mathrm{M}$ & IIC & T4bNOM0 & $3 / 3$ & $0 / 0$ & $3+$ & 2.00 & DOD/34 mos \\
\hline 18 & $45 / \mathrm{M}$ & IIB & T4aNOMO & $24 / 0$ & $0 / 0$ & $4+$ & 1.25 & DOD/53 mos \\
\hline 19 & $45 / F$ & IIA & T3aNOMO & $13 / 0$ & $0 / 0$ & $4+$ & 1.96 & AWD/54 mos \\
\hline 20 & $76 / \mathrm{M}$ & IIA & T3aNOMO & $13 / 0$ & $0 / 0$ & $4+$ & 1.08 & ANED/63 mos \\
\hline 21 & $55 / \mathrm{M}$ & $\| A$ & T3aNOMO & $12 / 0$ & $0 / 0$ & $2+$ & 0.13 & DUD/21 mos \\
\hline 22 & $51 / F$ & IIX & T3xNOMO & $12 / 0$ & $0 / 0$ & $2+$ & 0.38 & ANED/29 mos \\
\hline 23 & $54 / \mathrm{M}$ & IIX & T3xNOMO & $4 / 4$ & $0 / 0$ & $\mathrm{~N}$ & 0.00 & ANED/21 mos \\
\hline 24 & $26 / F$ & IIX & T3xNOMO & $2 / 2$ & $0 / 0$ & $2+$ & 1.25 & ANED/26 mos \\
\hline 25 & $35 / \mathrm{M}$ & $\mid A$ & T1aNOMO & $4 / 4$ & $0 / 0$ & $3+$ & 0.63 & ANED/21 mos \\
\hline 26 & $67 / \mathrm{M}$ & IX & $\mathrm{TxNOMO}^{b}$ & $1 / 1$ & $0 / 0$ & $\mathrm{~N}$ & 0.00 & ANED/22 mos \\
\hline
\end{tabular}

ANED, alive with no evidence of disease; AWD, alive with disease; DFOD, dead free of disease; DOD, dead of disease; DUD, dead of undetermined disease; IDO, indoleamine 2,3-dioxygenase; N, compatible with control; NH, highest node; NS, nodal score; S, satellite lesion; SNP, positive sentinel nodes; SNT, total sentinel nodes; TNM, local tumor growth (T), spread to regional lymph nodes (N), and distant metastases (M).

a Unknown primary.

${ }^{b}$ Subungual melanoma in situ with regression changes; destroyed overlying nail.

\section{Controls and Scoring System for IDO Immunostaining}

Initial evaluation of nodal IDO immunohistochemistry revealed that many lymph nodes from patients with melanoma harbored significant clusters of intensely positive IDO cells in the perisinusoidal and intrafollicular regions of the lymph node. Significant clusters were defined as confluent regions that demonstrated at least 20 IDO-positive cells per high power field $(\times 400)$ (Fig. 2A). In the final analysis, the perisinusoidal pattern of involvement (Fig. 2B) represented $97 \%$ of nodal involvement with IDO-positive cells, and we considered this to be the pathobiologic pattern.

Grading of these abnormal clusters involved determining the infiltration percentage of the entire perisinusoidal regions of any given node (normal $=<5 \% ; 1+=5 \%$ to $25 \% ; 2+=25 \%$ to $50 \% ; 3+=50 \%$ to $75 \% ; 4+=75 \%$ to $100 \%$ ). A study set was created, and using the above criteria, three pathologists (JRL, RRD, and JLM) independently graded 100 lymph nodes. There was agreement within one grade in $85 \%$ of cases. The IDO activity in each node dissection package was then evaluated by two parameters: the highest nodal grade was generated as the maximum grade of any node in the dissection packet and an IDO nodal score was computed by adding the grades of each reviewer and dividing by the number of grading events.

As controls, representing the normal pattern of IDOexpressing cells in lymphoid tissue, we used tonsillar tissue (removed from otherwise healthy pediatric patients for a diagnosis of symptomatic hypertrophy) and axillary lymph node dissections from patients with small locally confined breast cancer without subsequent metastasis. Of these controls, 27 were entirely negative without any chromogen deposition, while seven revealed only single scattered IDO-positive cells in the lymphoid regions (Fig. 2C). Two tonsils and one lymph node demonstrated isolated perisinusoidal clusters of IDOpositive cells, each comprising $<20$ cells per high power field, and on no occasion did the clustered activity involve $>5 \%$ of the perisinusoidal lymphoid regions. Thus, the $1+$ to $4+$ grading system did not overlap the range of normal in these samples, and all grades reflected abnormal accumulation of IDO-positive cells.

\section{Immunohistochemical Appearance of IDO-Positive DCs In Vivo}

Immunohistochemical staining of lymph nodes for IDO identified a characteristic population of cells that dem- 

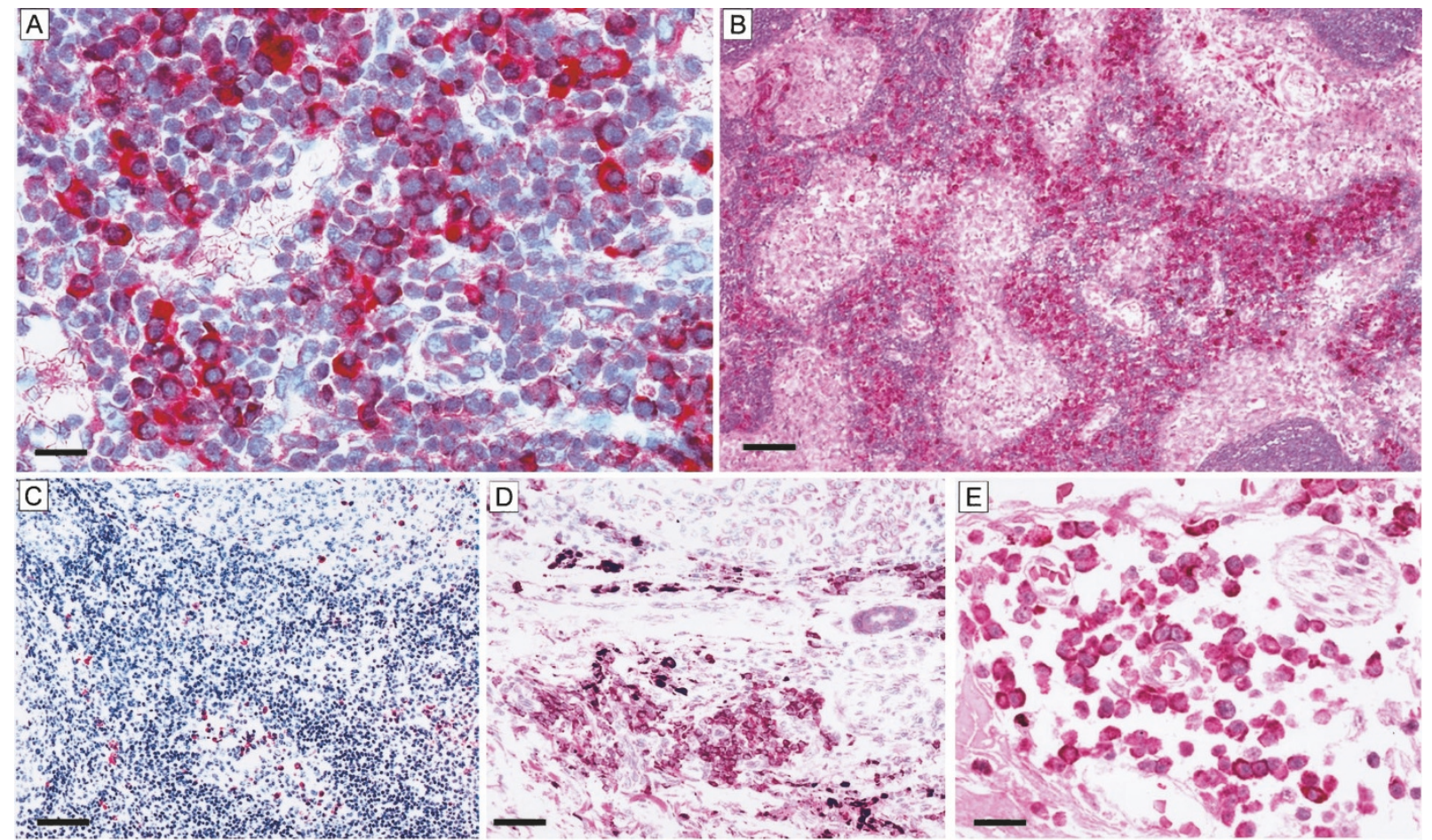

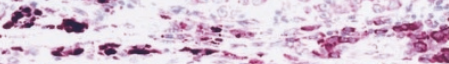
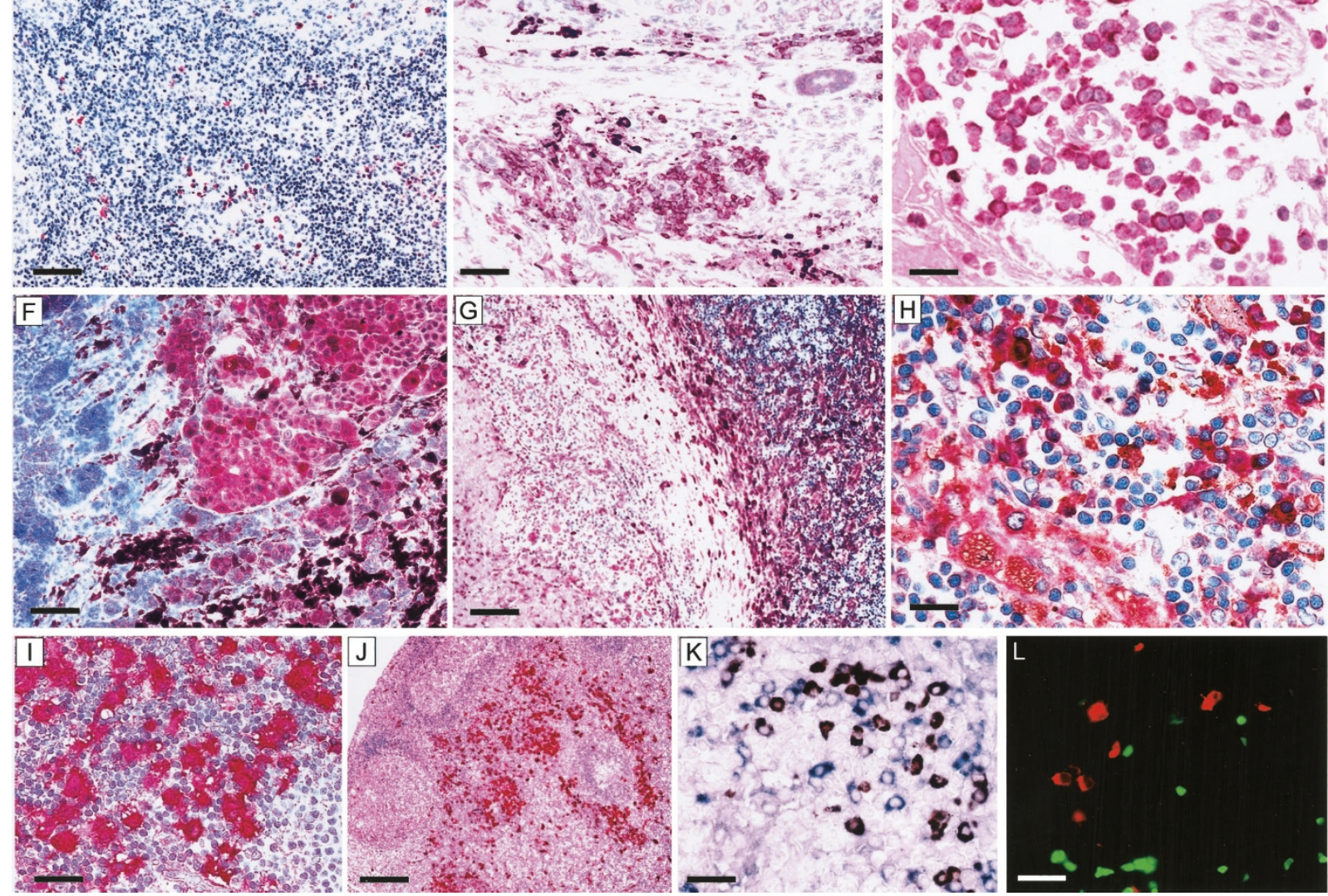

Figure 2.

Characteristics of tissue indoleamine 2,3-dioxygenase (IDO) immunostaining. (A) Clustering of red staining IDO-positive cells within a melanoma-draining lymph node. The cells demonstrate a monocytoid or plasmacytoid morphology, with eccentrically placed nuclei and scant granular cytoplasm. In areas, these cells mold around surrounding lymphocytes. Bar $=20 \mu \mathrm{m}$. (B) Distribution of red staining IDO-positive cells in the lymphoid regions (deep purple) adjacent to the central medullary sinuses (geographic pink regions harboring macrophages). Bar $=140 \mu \mathrm{m}$. (C) Control IDO staining of minimally immunostimulated human pharyngeal tonsil. Only scattered red staining IDO-positive cells are identified. Bar $=100 \mu \mathrm{m}$. (D) Collections of red staining IDO-positive cells in the inflammatory mantle (bottom) of a cutaneous melanoma primary (top). Dark brown melanin is seen in the interface tissue between tumor and underlying inflammatory response. Bar $=60 \mu \mathrm{m}$. (E) Perivascular cuffing by IDO-positive cells in inflammatory milieu of cutaneous primary. Small vessel with spiraling endothelial cells surrounded by red staining plasmacytoid cells. Cutaneous Pacinian corpuscle identified in upper-right corner. Bar $=36 \mu \mathrm{m}$. (F) Focal red IDO staining of melanoma tumor cells in a pattern suggestive of clonal expansion. Dark brown melanin identified at interface with IDO-negative tumor cells. Bar $=100 \mu \mathrm{m}$. (G) Red staining IDO-positive cells within the inflammatory mantle (center) of an advancing melanoma nodal metastasis (left). Note that the majority of IDO-positive cells intermingle with nodal lymphocytes (right). Bar $=50 \mu \mathrm{m}$. H) Dual immunostaining for IDO (red) and HAM56 (brown). The nature of the brown diaminobenzidine (Dako) immunostaining within the more abundant cytoplasm of the macrophages makes it impossible to rule out low-level co-expression of IDO in these cells. The intense ID0 staining, however, was confined to the plasmacytoid cells. Bar $=25 \mu \mathrm{m}$. (I) S-100 immunostaining of dendritic cells within melanoma-draining lymph node. The red staining is seen in larger cells that are heterogeneous in size and shape. These cells have abundant cytoplasm that extensively interdigitate with surrounding lymphocytes bar $=42 \mu \mathrm{m}$. J) Red staining S-100-positive dendritic cells occupy a peripheral perifollicular nodal location, in contrast to the central perisinusoidal distribution of ID0-positive cells. Bar $=350 \mu \mathrm{m}$. (K) Dual immunostaining for IDO (brown) and kappa/lambda light chains $(\kappa / \lambda)($ blue ) within a perisinusoidal region. Note that each differentially stained population of cells has a plasmacytoid appearance. Bar $=26 \mu \mathrm{m}$. (L) Dual immunofluorescence for IDO (red) and $\kappa / \lambda$ (green) confirms the presence of distinct cellular populations. Bar $=26 \mu \mathrm{m}$. 
onstrated intense cytoplasmic chromogen deposition. These cells had distinct cell borders and uniform, slightly eccentrically placed nuclei. They had scant minimally granular cytoplasm and were slightly larger than the surrounding lymphocytes. These cells were seen in close proximity to surrounding lymphocytes, and at times the IDO-positive cell cytoplasm molded around adjacent lymphocytes (Fig. 2A). In contrast, S-100 immunostaining recognized a conventional DC that was heterogeneous in size and shape (up to five times larger than the surrounding lymphocytes) and demonstrated eccentrically placed nuclei of differing size and shape with small nucleoli. There was abundant cytoplasm with slight granularity. There was extensive interdigitation of the cytoplasm around surrounding lymphocytes (Fig. 2l).

Infiltration of IDO-positive cells was identified within malignant melanoma lymph node dissections. In many nodes that harbored metastatic disease, clusters of IDO-positive cells were found in the peritumoral inflammatory infiltrate (Fig. 2G). Importantly, however, there were many cases in which the nodes were negative for any detectable malignancy, yet there were clearly abnormal collections of IDO-positive cells. The majority of the IDO-positive cell clusters were found intermingled with lymphocytes closely adjacent to nodal sinusoids (Fig. 2B). A few nodes demonstrated clusters of IDO-positive cells within follicles or in clusters surrounding high-endothelial venules. In contrast, the conventional (S-100 positive) DCs were found to localize primarily in the peripheral perifollicular lymphocyte-bearing regions, and spared the lymphoid tissues surrounding the medullary sinuses (Fig. 2J). Dual staining for IDO and the macrophage markers HAM56 and CD68 revealed nonoverlapping populations of sinus histiocytes and IDO-positive cells (Fig. 2H). Thus, the IDO-positive cells were a distinct population that were neither conventional interdigitating DCs nor macrophages.

Because many IDO-positive cells demonstrated a histologic appearance similar to plasma cells, dual staining for IDO and the light chains kappa and lambda was performed. Immunohistochemistry and dual-color immunofluorescence confirmed the existence of two cell populations within the perisinusoidal regions (Fig. 2, $\mathrm{K}$ and $\mathrm{L}$ ). The majority of cells with conventional plasma cell morphology were $\kappa / \lambda$ positive, consistent with terminally differentiated $B$ cells. The majority of IDO-positive cells were $\kappa / \lambda$ negative by immunofluorescence, and thus were entirely distinct from plasma cells. Dual immunofluoresence also revealed scattered cells that appeared to demonstrate co-localization of IDO and $\kappa / \lambda$ staining. This might represent authentic staining of a minor subset of B-cell lineage cells, although we could not rule out nonspecific binding to Fc receptors or other artifact. However, the IDOpositive and $\kappa / \lambda$-negative cells comprised an unambiguous population, which were distinct from B-lineage cells (Fig. 2L).

\section{IDO Expression in Lymph Node Dissections}

For quantitative grading, two pathologists (JRL and RRD) graded all study nodes from the patients. Any node that demonstrated sufficient residual lymph node architecture, regardless of presence or absence of metastatic disease, was graded. Of the 328 lymph nodes examined, 22 showed complete tumoreffacement (with insufficient central medullary sinuses), and these were excluded from the scoring group. Seventy-three percent of these 22 effaced nodes did, however, demonstrate an infiltration of IDO-positive cells at the advancing tumor border, similar to the reaction to cutaneous primaries and cutaneous metastases (Fig. 2, D and G). There were 29 lymph nodes that harbored small metastases and had sufficient residual lymph node architecture to allow IDO grading in the perisinusoidal regions. Of these only two cases (7\%) revealed focal IDO-positive cellular infiltration at the tumor-lymph node interface. Focal IDO staining within tumor cells was seen in 6 of the 51 nodal malignant deposits (effaced and noneffaced), and this staining was similar to the tumor staining seen in cutaneous primaries (Fig. 2F).

Of the 306 lymph nodes that were scored, 55\% demonstrated limited immunohistochemical staining that was identical to the control tissue, consisting of 14 minimally hyperplastic tonsils and 23 lymph nodes from early stage, node-negative breast cancers. Further, $17 \%$ of scored lymph nodes were $1+, 12 \%$ were $2+, 9 \%$ were $3+$, and $7 \%$ were $4+$. Thirteen of 26 $(50 \%)$ patients demonstrated at least one lymph node with greater than half of the perisinusoidal regions infiltrated with significant clusters of IDO-positive cells $(>3+)$. As a result, it became evident that lymph nodes draining malignant melanoma, with or without metastatic disease, were clearly different from control lymphoid tissues. Statistical analysis of these two populations confirmed this diversity $(p<0.001)$.

\section{IDO Expression in Primary Cutaneous Tumors}

Thirteen primary tumors associated with the study lymph node dissections were available for IDO immunostaining. In addition there were four blocks of cutaneous melanoma metastases from one patient. In seven cases (including all metastases), IDO-positive cells were identified in the inflammatory mantle at the advancing edge of tumor (Fig. 2D). Scattered cells were seen in and among the recruited lymphocytes, but the majority of cells were perivascular (Fig. 2E). In pigmented tumors there was nonspecific deposition of chromogen associated with melanin, and IDO immunostaining of focal nests of tumor cells was seen in two cases (Fig. 2F). In addition, nonspecific chromogen deposition was seen associated with melanin of dermal melanophages, obscuring the ability to identify cytoplasmic IDO staining in these cells. There was also a variable degree of diffuse pale cytoplasmic IDO staining within surrounding epidermal keratinocytes and eccrine glandular epithelium. 


\section{Expression of Macrophage Inflammatory Protein (Mip)-3 $\alpha$ by Tumors}

To understand the apparent preferential accumulation of IDO-expressing cells in tumor-draining lymph nodes, we speculated that the tumors secrete a factor that selectively attracted these cells. We knew from our in vitro model (Fig. 1) that the chemokine receptor CCR6 co-segregated with IDO expression in monocyte-derived DCs. We therefore asked whether tumors might produce a ligand for CCR6. Mip- $3 \alpha$ is the only known chemokine ligand for CCR6 (Liao et al, 1999), and it has been reported to be highly expressed in inflamed human tonsils (Dieu et al, 1998), where we have previously demonstrated accumulations of IDOpositive cells (Munn et al, 2002). As a more specific prediction, mip- $3 \alpha$ is also expressed in the subepithelial tissues overlying small intestinal mucosal lymphoid aggregates in mice (Iwasaki and Kelsall, 2000). DCs expressing CCR6 are recruited into this region by mip-3 $\alpha$, and mice lacking the CCR6 receptor fail to recruit a population of DCs into this specific subepithelial region of the gut (Varona et al, 2001). Therefore, based on these data in the murine models, we examined the analogous anatomic region in human intestines to test the prediction that IDO-positive cells would be found to co-localize with regions of mip-3 $\alpha$ expression. By dual-label immunohistochemistry, we observed focal collections of cells that stained
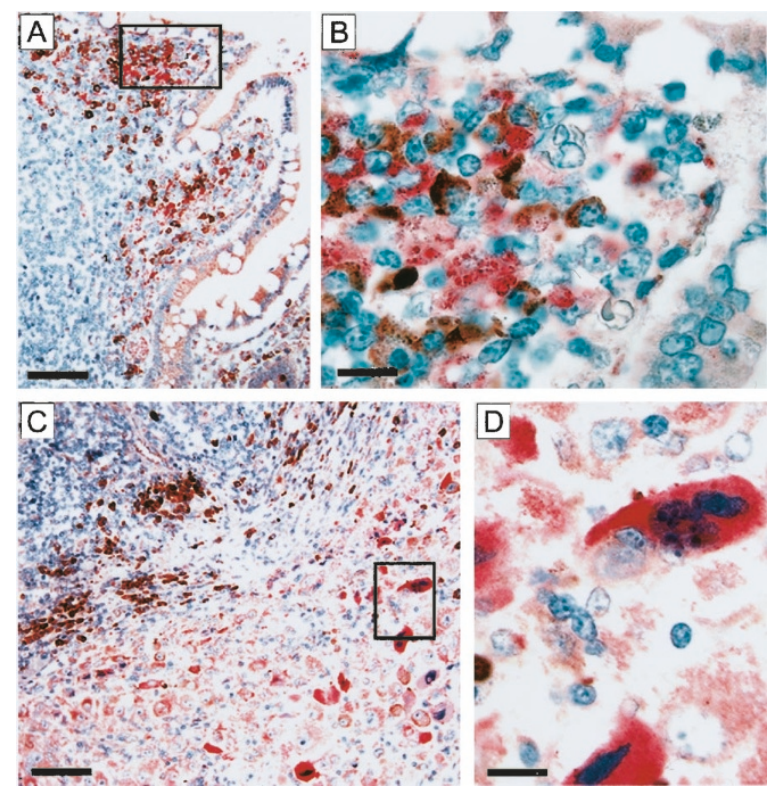

Figure 3.

Indoleamine 2,3-dioxygenase (IDO) and macrophage inflammatory protein (mip)-3 $\alpha$ immunostaining. (A) Co-existence of cells expressing IDO (brown) and mip- $3 \alpha$ (red) in the lamina propria of the small intestine, particularly in the subepithelial areas overlying mucosal lymphoid aggregates. Bar $=50 \mu \mathrm{m}$. (B) Higher magnification of the indicated region in panel A. Note that the dual population of cells are intermingling with lamina propria lymphocytes. Bar = $16 \mu \mathrm{m}$. (C) Expression of mip-3 $\alpha$ (red) by tumor cells (lower right) of nodal metastatic melanoma. ID0-positive cells are congregated with lymphocytes at the advancing margin of the metastasis (upper left). Bar $=50 \mu \mathrm{m}$. (D) Higher magnification of the indicated region in panel $\mathrm{C}$ that demonstrates intense red mip-3 $\alpha$ staining within a multinucleated malignant cell. Bar $=16 \mu \mathrm{m}$. strongly for IDO, in close apposition to cells expressing mip-3 $\alpha$ (Fig. 3, A and B). Dual staining of a lymph node harboring metastatic melanoma demonstrated expression of mip- $3 \alpha$ within tumor cells and localized aggregation of IDO-positive cells (Fig. 3, C and D).

Based on these descriptive data, we next asked whether human tumors produced mip- $3 \alpha$. At the time of resection, random biopsy specimens were obtained from melanomas, renal cell carcinomas, and nonsmall cell pulmonary carcinomas, and RNA was extracted (a single random biopsy was not necessarily representative of the entire tumor, and thus might underestimate the number of tumors expressing mip-3 $\alpha$ somewhere in the tumor mass, but a positive result would be a true positive). Figure 4 shows that analysis of mip- $3 \alpha$ mRNA by reverse transcriptase and real-time PCR demonstrated expression of mip- $3 \alpha$ in a significant number of melanomas and other tumor types, even in a single random biopsy. Thus, our data were not inconsistent with the hypothesis that mip- $3 \alpha$ might help recruit IDO-expressing cells to tumordraining lymph nodes.

\section{Discussion}

Early in development, tumors are able to elude innate host immunosurveillance, in spite of expressing unique tumor-associated antigens. This tumor immunotolerance can arise passively due to a lack of tumor interaction with the immune system (Ochsenbein et al, 2001) or as an active antigen-driven process of T-cell alteration. In a seminal paper, Staveley-O'Carroll and colleagues (1998) demonstrated that tumors could actively induce immunotolerance. This group further demonstrated that T-cell anergy was not caused by interaction with tumor cells, but by cross presentation

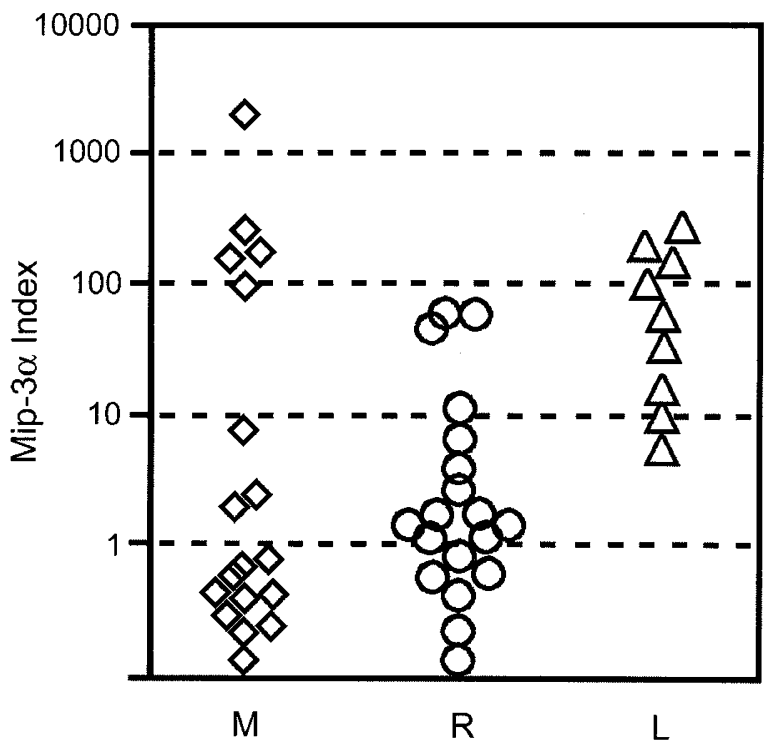

Figure 4.

Expression of macrophage inflammatory protein (mip)-3 $\alpha$ mRNA by human tumors. RNA from melanomas $(M)$, renal cell carcinomas $(R)$, or non-small cell lung carcinomas $(L)$ was analyzed for expression of mip-3 $\alpha$ by reverse transcriptase real-time quantitative PCR. Data represents a ratio of mip-3 $\alpha$ to glyceraldehyde-3-phosphate dehydrogenase, with control line set to equal 1. 
of tumor antigens by tolerogenic bone marrow-derived APCs (Sotomayor et al, 2001). In humans, it has been shown that injection of antigen pulsed immature myeloid DCs can inhibit antigen-specific CD8 ${ }^{+}$effector T cells (Dhodapkar et al, 2001). The development of immune tolerance not only involves T-cell anergy or deletion but may also involve the emergence of a population of regulatory T cells (Jonuleit et al, 2000). There is strong evidence that APCs are key in inducing and modulating these cells that are critically involved in maintenance of self-tolerance (Liu, 2001) and tumor immunotolerance (Smyth et al, 2001).

Immature DCs were originally considered to be responsible for tolerance induction of $T$ cells. These immature DCs express lower levels of major histocompatibility complex molecules and lack the expression of co-stimulatory molecules at the time of antigen presentation. There is evidence, however, that mature APCs can also induce tolerance (Albert et al, 2001; Menges et al, 2002). We have shown that IDOexpressing APCs, both immature and mature, inhibit antigen-driven proliferation of lymphocytes in mixedleukocyte reactions (Munn et al, 2002). We speculate that IDO-positive cells in tumor-draining lymph nodes may be both effective antigen-presenting cells and also be contributors to immune tolerance.

To date, in vivo evaluation reveals that IDO can be expressed by a variety of cell types. In the immune system, this can include subsets of DCs, T cells (Curreli et al, 2001), and possibly a subset of terminally differentiated B cells. In this study we show that IDO-positive cells accumulate in an abnormal fashion in a selected number of lymph nodes draining malignant melanoma. Recent evidence suggests that presentation of tumor antigens to $T$ cells takes place in the draining lymph nodes (Ochsenbein et al, 2001). We hypothesize that this is biologically significant, because depending on the type of antigen-presenting cell and the cytokine milieu, the immune response to tumor antigens may be pathologic tolerance or anergy. In this regard, it is notable that even sentinel nodes without metastatic involvement could demonstrate a markedly abnormal accumulation of IDOpositive cells. We hypothesize that these nodal IDOpositive cells are recruited in response to tumorinduced changes in the tumor-draining lymph node and that these cells present tumor-derived antigens in a fashion that leads to immunologic unresponsiveness. In view of the fact that we find CCR6 to be preferentially expressed on IDO-positive DCs and its ligand is expressed by many tumors, we propose that this mechanism may contribute to this recruitment of IDO-positive DCs to tumor-draining lymph nodes.

Cochran and colleagues (2001) have reported that nonmalignant sentinel nodes demonstrated significant architectural and cellular changes as compared with downstream benign nonsentinel nodes, possibly from the effects of tumor-derived antigens. They showed that there was a dramatic decrease in paracortical IDCs in association with a reduction in the thickness of the paracortical regions. They hypothesized that these malignant melanoma-draining lymph nodes are primed for immune tolerance prior to the appearance of metastases. As a corollary, we feel that IDOpositive cells found in downstream draining lymph nodes may represent participation in this initiation and propagation of tumor immune tolerance.

The small number of nodal dissections and the heterogeneity of sentinel and nonsentinel nodes limited our ability to perform meaningful statistical analysis. However, the large number of IDO-positive cells in tumor-draining nodes compared with controls was clearly abnormal and highly statistically significant. This finding combined with the biologic ability of IDO-expressing cells to inhibit immune responses warrants further large-scale studies to assess the predictive value of IDO expression in melanoma. Indeed, a collaborative study is underway to confirm the prognostic utility of IDO immunostaining in melanoma sentinel lymph nodes. In addition, if melanomas are able to recruit increased numbers of immunosuppressive IDO-positive DCs into tumor-draining lymph nodes, then pharmacological blockade of this mechanism may prove important in therapeutics.

\section{Materials and Methods}

\section{Culture and Flow Cytometry}

Human monocytes (> 95\% purity) were isolated by leukocytapheresis and counterflow elutriation (Munn et al, 1999) under a protocol approved by our institutional review board. To generate DCs, monocytes were cultured for 7 days in serum-free X-vivo 15 medium (BioWhitaker) supplemented with granulocyte-macrophage colony-stimulating factor (50 ng/ml, R\&D Systems, Minneapolis, Minnesota) + IL4 (50 ng/ml, R\&D Systems) on Days 0, 2, and 4. Mature DCs were generated by adding the following cocktail (Jonuleit et al, 1997) during the final 48 hours of culture: TNF $\alpha(1100 \mathrm{U} / \mathrm{ml}$, BD-Pharmingen, San Diego, California), IL1 $\beta$ (1870 U/ml, BD-Pharmingen), IL6 (1000 U/ml, BD-Pharmingen), and PGE2 (1 ug/ml, Sigma, St. Louis, Missouri).

For IDO staining, nonadherent DCs and adherent cells (harvested with EDTA) were pooled stained with anti-CD123-biotin (clone 7G3, BD-Pharmingen) followed by streptavidin-perCP, and anti-CD11callophycocyanin (BD-Pharmingen). After fixation and permeablization (Cytofix/Cytoperm, BD-Pharmingen), cells were stained with rabbit anti-IDO antibody (Chemicon, Temecula, California) followed by PElabeled anti-rabbit secondary antibody (Jackson Immunoreasearch, West Grove, Pennsylvania), crossadsorbed against mouse, human, and bovine IgG. For all experiments, the negative control for IDO staining was the anti-IDO antibody pre-adsorbed with a 50fold molar excess of the immunizing peptide. DCs were gated on forward and side scatter to exclude contaminating lymphocytes and debris. For phenotyping experiments, cells were stained without permeablization using a multicolor panel of CD123 (to identify the IDO-positive subset) versus various DC markers (all from BD-Pharmingen). Our DC culture 
conditions were optimized to support myeloid DC differentiation. In this system, the lymphoid population of plasmacytoid DCs, defined as CD123 positive and CD11c negative, was undetectable after 7 days of culture.

\section{Patient Samples}

Twenty-six cases of malignant melanoma patients requiring lymph node dissection were compiled from the archives of the Medical College of Georgia and the Augusta Veterans Affairs Medical Center from 1990 to 2000. Patients with complete nodal dissections and patients with sentinel lymphadenectomy were included. A total of 328 regional lymph nodes, including 22 sentinel nodes, were accrued. In addition, 14 paraffin blocks of cutaneous melanoma (13 primary tumors and one with cutaneous metastases), all associated with the study lymph node dissections, were available for immunohistochemical evaluation.

\section{Immunohistochemistry}

Tissue Immunohistochemistry: Following procurement of formalin-fixed paraffin-embedded blocks under a protocol approval by our institutional review board, all cases were evaluated with $5-\mu \mathrm{m}$ replicate sections stained with hematoxylin and eosin. Immunohistochemistry was performed after xylene deparaffinization using the standard avidin-biotin complex method. Affinity-purified polyclonal rabbit antibodies were used to immunostain for IDO (Munn et al, 2002). In addition, rabbit polyclonal S-100 (DAKO Corporation, Carpenteria, California), mouse monoclonal light chain kappa (DAKO), mouse monoclonal light chain lambda (DAKO), mouse CD68 (PG-M1, DAKO), mouse HAM56 (DAKO), and polyclonal goat mip-3 $\alpha$ (R\&D Systems) were also used. Pretreatment with proteinase $\mathrm{K}$ (DAKO) was performed before immunostaining for IDO, S-100, kappa, lambda, CD68, and HAM56. Antigen retrieval with citrate (Target, DAKO) was performed prior to immunostaining for mip-3 $\alpha$. Detection was obtained using species-specific secondary antibody conjugated to alkaline phosphatase (LSABrabbit kit, DAKO) or horseradish peroxidase (HRP) (LSAB2, DAKO). Chromogen development was performed with Fast Red (Vector Laboratories, Burlingame, California) or diaminobenzidine (DAB, DAKO).

For dual immunostaining of IDO versus kappa/ lambda $(\kappa / \lambda)$, the $\kappa / \lambda$ monoclonal antibodies were mixed and applied to deparaffinized slides following proteinase $\mathrm{K}$ pretreatment. Detection was performed using species-specific secondary antibody conjugated to alkaline phosphatase with nitro-blue tetrazolium chromogen (DAKO). Following incubation with the IDO antibody, detection was performed with a species-specific HRP conjugated secondary antibody with 3-amino-9-ethylcarbazole chromogen (DAKO). For all other dual immunostaining, the first antibody was applied following appropriate antigen retrieval and detected with HRP/DAB. The slides were then subjected to additional antigen retrieval, if necessary, and stained for the second antigen using alkaline phosphatase/Fast Red for detection.

Dual Immunofluorescence: For IDO versus $\kappa / \lambda$ dual immunofluorescence, deparaffinized $5-\mu \mathrm{m}$ replicate sections were subjected to proteinase K pretreatment. Following primary antibody incubation, detection was performed using species-specific secondary antibodies conjugated to either Alexa-488 or Alexa-568 (Molecular Probes, Eugene, Oregon).

IDO Immunohistochemical Controls: To assess baseline IDO immunostaining controls, lymphoid tissues that we predicted to have minimal immunostimulation were selected and stained. These included 14 minimally hyperplastic tonsil specimens in young patients and 23 lymph nodes from six patients with early-stage node-negative breast cancer. Positive and negative controls were performed on all stained sections.

\section{Reverse Transcriptase PCR}

RNA from 18 melanomas, 19 renal cell carcinomas, and 9 non-small cell lung cancers were obtained from the H. Lee Moffitt Cancer Center and Research Institute tissue procurement laboratory under a protocol approved by the Moffitt Cancer Center institutional review board. cDNA was synthesized from $1 \mu \mathrm{g}$ of total cellular RNA by reverse transcription with Sensiscript reverse transcriptase (Qiagen, Valencia, California) using random hexamers as primers. The cDNA was then analyzed using the LightCycler real-time PCR system (Roche) and FastStart DNA Amplification Kit (SYBR Green 1, Roche). The primers used were: glyceraldehyde-3-phosphate dehydrogenase (GenBank Gl:7669491, sense b.p. 87-104, antisense b.p. 289-307), and mip-3 $\alpha$ (GenBank Gl:4759075, sense b.p. 103-121, antisense b.p 410-428). Standard curves were prepared from U937 cells induced with phorbol myristate acetate for 24 hours and were linear $(r=-0.99)$ in the range of $100 \mathrm{pg}$ to $100 \mathrm{ng}$ total RNA. These primers yielded a PCR product that was a single band by gel electrophoresis, and the standard curve was linear $(r=-0.99)$ over the range of 0.01 to $100 \mathrm{pg}$ total RNA. To allow comparison between different samples, the data were presented as the ratio of mip-3 $\alpha$ to the glyceraldehyde-3-phosphate dehydrogenase housekeeping gene in each sample (arbitrary units). The ratio in the control cell line (resting U937 cells) was set equal to 1 .

\section{Statistics}

Fisher's exact test (two-tailed) was used to compare the IDO immunostaining between the control group and the melanoma lymph node group.

\section{Acknowledgements}

The authors wish to thank Drs. James and Mary O'Quinn for contribution of cases and to Hemal Patel and Amber Gardner for their assistance in this project. 


\section{References}

Albert ML, Jegathesan M, and Darnell RB (2001). Dendritic cell maturation is required for the cross-tolerization of $\mathrm{CD}^{+}$ T cells. Nat Immunol 2:1010-1017.

Balch CM, Buzaid AC, Soong S-J, Atkins MB, Cascinelli N, Coit DG, Fleming ID, Gershenwald JE, Houghton A, Kirkwood JM, McMaster KM, Mihm MF, Morton DL, Reintgen DS, Ross MI, Sober A, Thompson JA, and Thompson JF (2001). Final version of the American Joint Committee on Cancer staging system for cutaneous melanoma. J Clin Oncol 19:36353648.

Bowne WB, Srinivasan R, Wolchok JD, Hawkins WG, Blachere NE, Dyall R, Lewis JJ, and Houghton AN (1999). Coupling and uncoupling of tumor immunity and autoimmunity. J Exp Med 190:1717-1722.

Cochran AJ, Morton DL, Stern S, Lana AMA, Essner R, and Wen D-R (2001). Sentinel lymph nodes show profound downregulation of antigen-presenting cells of the paracortex: Implications for tumor biology and treatment. Mod Pathol 14:604-608.

Curreli S, Romerio F, Mirandola P, Barion P, Bemis K, and Zella D (2001). Human primary $\mathrm{CD}^{+}{ }^{+} \mathrm{T}$ cells activated in the presence of IFN- $\alpha 2 b$ express functional indoleamine 2, 3-dioxygenase. J Interferon Cytokine Res 21:431-437.

Dhodapkar MV, Steinman RM, Krasovsky J, Munz C, and Bhardwaj N (2001). Antigen-specific inhibition of effector $T$ cell function in humans after injection of immature dendritic cells. J Exp Med 193:233-238.

Dieu MC, Vanbervliet B, Vicari A, Bridon JM, Oldham E, Ait-Yahia S, Briere F, Zlotnik A, Lebecque S, and Caux C (1998). Selective recruitment of immature and mature dendritic cells by distinct chemokines expressed in different anatomic sites. J Exp Med 188:373-386.

Doan T, Herd KA, Lambert PF, Fernando GJ, Street MD, and Tindle RW (2000). Peripheral tolerance to human papillomavirus E7 oncoprotein occurs by cross-tolerization, is largely Th-2-independent, and is broken by dendritic cell immunization. Cancer Res 60:2810-2815.

Grohmann U, Fallarino F, Bianchi R, Belladonna ML, Vacca C, Orabona C, Uyttenhove C, Fioretti MC, and Puccetti $P$ (2001). IL-6 inhibits the tolerogenic function of CD8alpha(+) dendritic cells expressing indoleamine 2, 3-dioxygenase. $\mathrm{J}$ Immunol 167:708-714.

Grohmann U, Orabona C, Fallarino F, Vacca C, Calcinaro F, Falorni A, Candeloro P, Belladonna ML, Bianchi R, Fioretti MC, and Puccetti $P$ (2002). CTLA-4-Ig regulates tryptophan catabolism in vivo. Nat Immunol 3:1097-1101.

Houghton AN, Gold JS, and Blachere NE (2001). Immunity against cancer: Lessons learned from melanoma. Curr Opin Immunol 13:134-140.

Hwu P, Du MX, Lapointe R, Do M, Taylor MW, and Young HA (2000). Indoleamine 2, 3-dioxygenase production by human dendritic cells results in the inhibition of $\mathrm{T}$ cell proliferation. J Immunol 164:3596-3599.

Iwasaki A and Kelsall BL (2000). Localization of distinct Peyer's patch dendritic cell subsets and their recruitment by chemokines macrophage inflammatory protein (MIP)-3alpha, MIP-3beta, and secondary lymphoid organ chemokine. J Exp Med 191:1381-1394.
Jonuleit H, Kuhn U, Muller G, Steinbrink K, Paragnik L, Schmitt E, Knop J, and Enk AH (1997). Pro-inflammatory cytokines and prostaglandins induce maturation of potent immunostimulatory dendritic cells under fetal calf serum-free conditions. Eur J Immunol 27:3135-3142.

Jonuleit H, Schmitt E, Schuler G, Knop J, and Enk AH (2000). Induction of interleukin 10-producing, nonproliferating CD4(+) T cells with regulatory properties by repetitive stimulation with allogeneic immature human dendritic cells. J Exp Med 192:1213-1222.

Jonuleit H, Schmitt E, Steinbrink K, and Enk AH (2001). Dendritic cells as a tool to induce anergic and regulatory $\mathrm{T}$ cells. Trends Immunol 22:394-400.

Klein C, Bueler H, and Mulligan RC (2000). Comparative analysis of genetically modified dendritic cells and tumor cells as therapeutic cancer vaccines. J Exp Med 191:16991708.

Liao F, Rabin RL, Smith CS, Sharma G, Nutman TB, and Farber JM (1999). CC-chemokine receptor 6 is expressed on diverse memory subsets of $T$ cells and determines responsiveness to macrophage inflammatory protein $3 \alpha$. J Immunol 162:186-194.

Liu YJ (2001). Dendritic cell subsets and lineages, and their functions in innate and adaptive immunity. Cell 106:259-262.

Mellor AL, Keskin DB, Johnson T, Chandler P, and Munn DH (2002). Cells expressing indoleamine 2, 3-dioxygenase inhibit T cell responses. J Immunol 168:3771-3776.

Menges M, Rößner S, Voigtländer C, Schindler H, Kukutsch MA, Bogdan C, Erb K, Schuler G, and Lutz MB (2002). Repetitive injection of dendritic cells matured with tumor necrosis factor $\alpha$ induce antigen-specific protection of mice from autoimmunity. J Exp Med 195:15-21.

Munn DH, Shafizadeh E, Attwood JT, Bondarev I, Pashine A, and Mellor AL (1999). Inhibition of T cell proliferation by macrophage tryptophan catabolism. J Exp Med 189:13631372.

Munn DH, Sharma MD, Lee JR, Jhaver KG, Johnson TS, Keskin DB, Marshall B, Chandler P, Antonia SJ, Burgess R, Slingluff CL, and Mellor AL (2002). Potential regulatory function of human dendritic cells expressing indoleamime 2, 3-dioxyenase. Science 297:1867-1870.

Munn DH, Zhou M, Attwood JT, Bondarev I, Conway SJ, Marshall B, Brown C, and Mellor AL (1998). Prevention of allogeneic fetal rejection by tryptophan catabolism. Science 281:1191-1193.

Ochsenbein AF, Sierro S, Odermatt B, Pericin M, Karrer U, Hermans J, Hemmi S, Hengartner $\mathrm{H}$, and Zinkernagel RM (2001). Roles of tumour localization, second signals and cross priming in cytotoxic T-cell induction. Nature 411:10581064.

Pardoll D (2001). T cells and tumours. Nature 411:10101012.

Shimizu K, Thomas EK, Giedlin M, and Mule JJ (2001). Enhancement of tumor lysate- and peptide-pulsed dendritic cell-based vaccines by the addition of foreign helper protein. Cancer Res 61:2618-2624.

Smyth MJ, Godfrey DI, and Trapani JA (2001). A fresh look at tumor immunosurveillance and immunotherapy. Nat Immunol 2:293-299. 
Sotomayor EM, Borrello I, Rattis FM, Cuenca AG, Abrams J, Staveley-O'Carroll K, and Levitsky HI (2001). Crosspresentation of tumor antigens by bone marrow-derived antigen-presenting cells is the dominant mechanism in the induction of T-cell tolerance during B-cell lymphoma progression. Blood 98:1070-1077.

Staveley-O'Carroll K, Sotomayor E, Montgomery J, Borrello I, Hwang L, Fein S, Pardoll D, and Levitsky H (1998). Induction of antigen-specific T cell anergy: An early event in the course of tumor progression. Proc Natl Acad Sci USA 95:11781183.
Steinman RM and Nussenzwieg MC (2002). Avoiding horror autotoxicus: The importance of dendritic cells in peripheral T cell tolerance. Proc Natl Acad Sci USA 99:351-358.

Varona R, Villares R, Carramolino L, Goya Í, Zaballos Á, Gutiérrez J, Torres M, Martínez-A C, Márquez G (2001). CCR6-deficient mice have impaired leukocyte homeostasis and altered contact hypersensitivity and delayed-type hypersensitivity responses. J Clin Invest 107:R37-R45. 